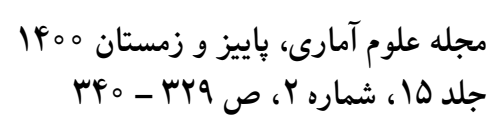

DOI: $10.29252 /$ jss.15.2.329

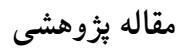

ساخت مدل بِيشكوى نايارامترى براى ميدان فضايى با استفاده از قضيه تصوير

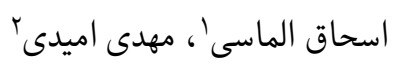

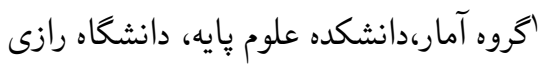

r كروه رياضى، دانشكده علوم بايه، دانشكاه ايلام

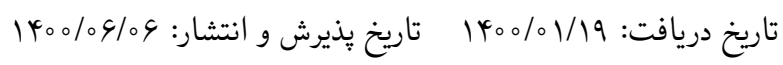

جكيده: تعيين بهترين بيشكوى فضايى براى مقادير كمشده يكى از مسائل مهم در آمار فضايى بهشمار

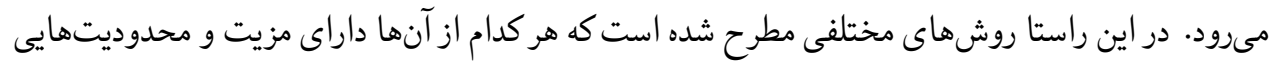

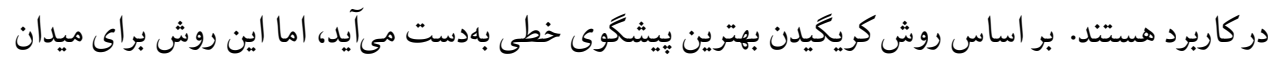

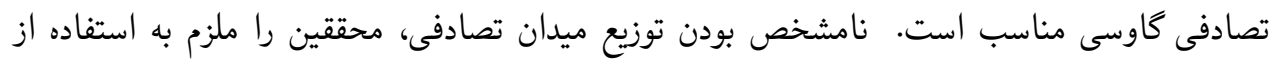

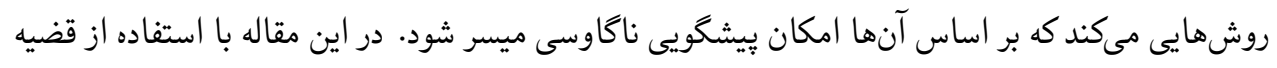

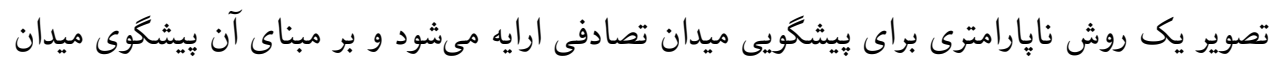

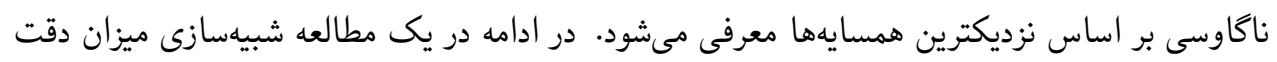

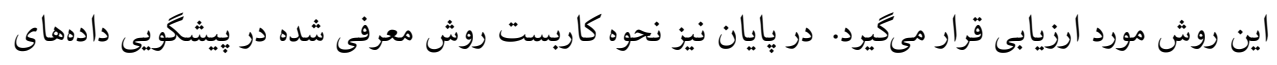
بارندكى در استان خوزستان نشان داده مىشود. وازمهاى كليدى: بيشكويى ناكاوسى، ميدان تصادفى، كريخيدن، قضيه تصوير. 
مدل بيشكوى نايارامترى فضايى با استفاده از قضيه تصوير

ro

\section{|}

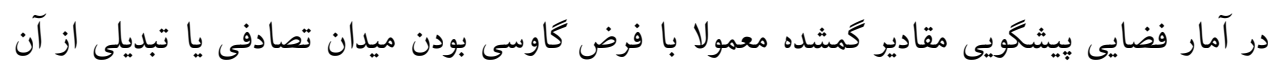

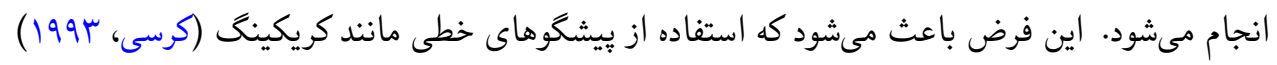

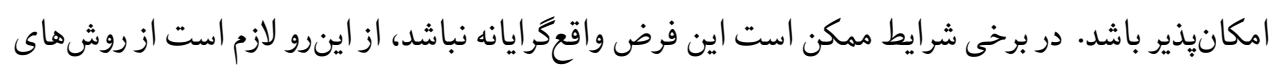

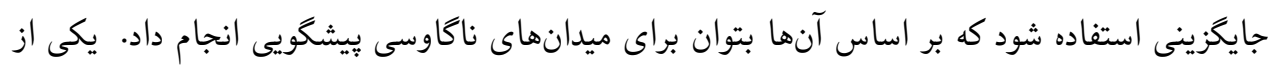

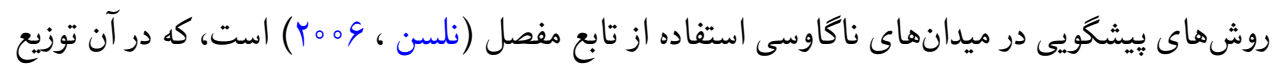

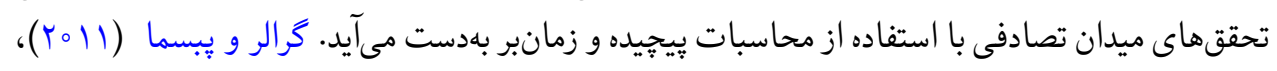

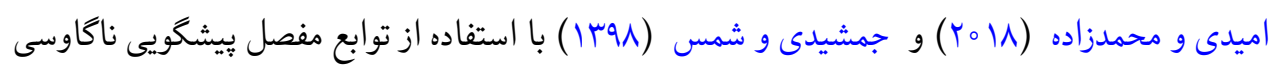

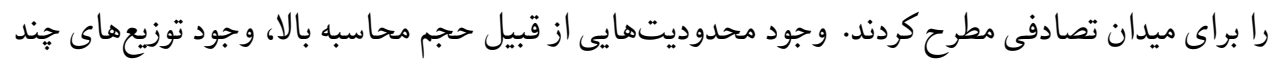

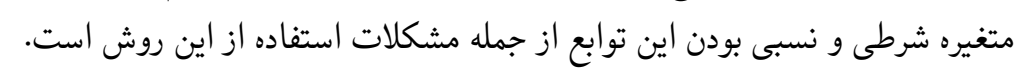

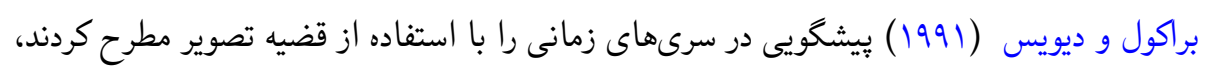

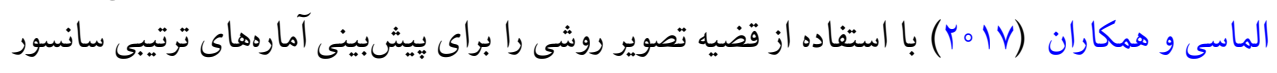

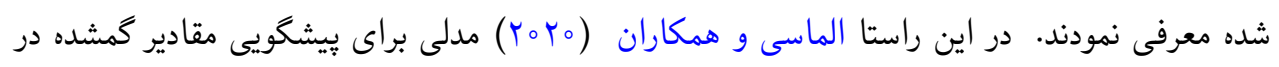

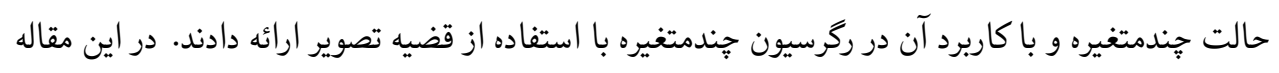

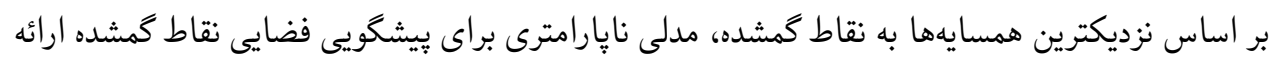

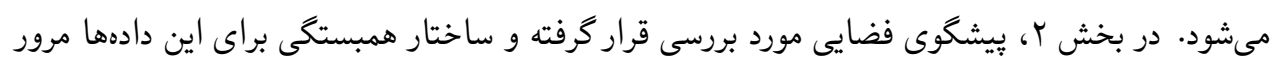

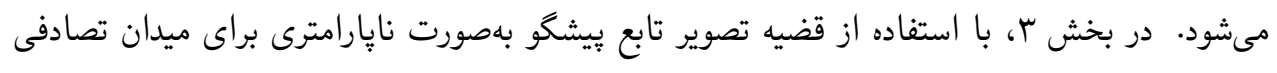

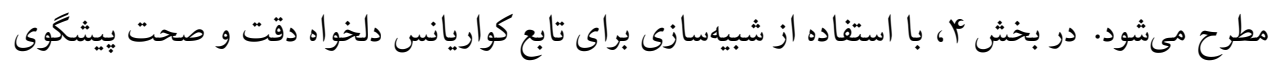

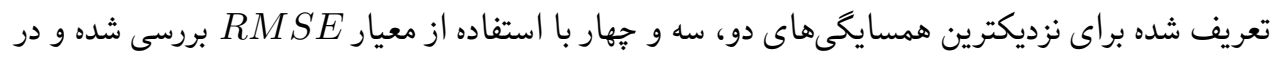

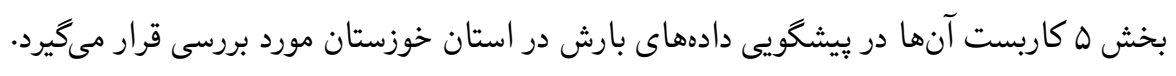

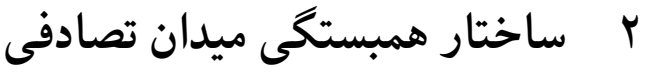

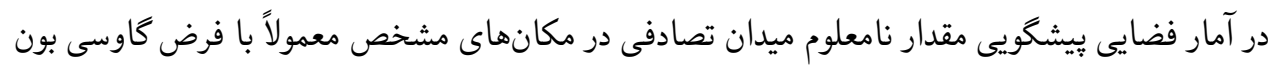

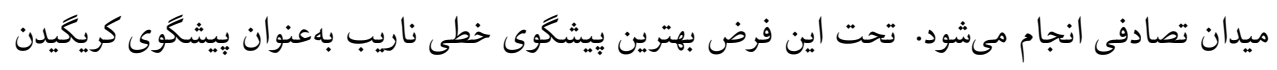
بهدست مىآيد. توابع ميانكين و كواريانس فضايى ميدان تصادفى 


$$
\begin{aligned}
\mu(s) & =E(Z(s)) ; \quad s \in D \\
C\left(s, s^{\prime}\right) & =\operatorname{Cov}\left(Z(s), Z\left(s^{\prime}\right) ; \quad s, s^{\prime} \in D\right.
\end{aligned}
$$

تعريف مىشوند. ميدان تصادفى (·)

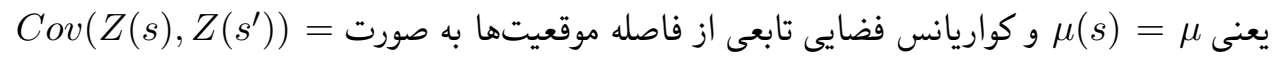
C باشد، كه در آن 'h

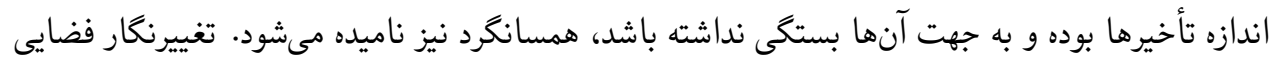

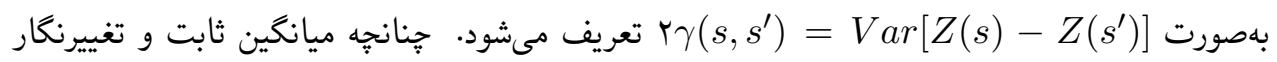
تابعى از تأخير باشد، يعنى (s)

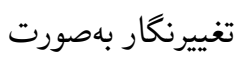

$$
r \gamma(h)=r[C(\circ)-C(h)]
$$

با تابع كواريانس فضايى در ارتباط است. توابع كواريانس فضايى ساختار همبستخى دادهاى فضايى را

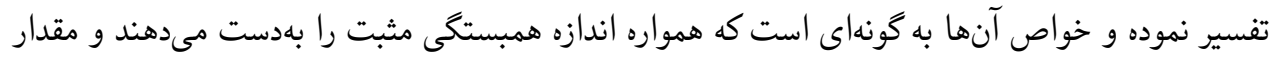

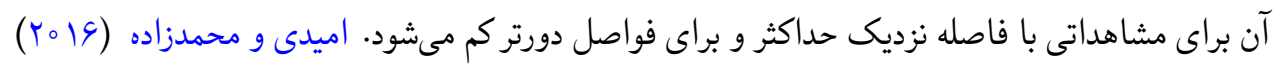

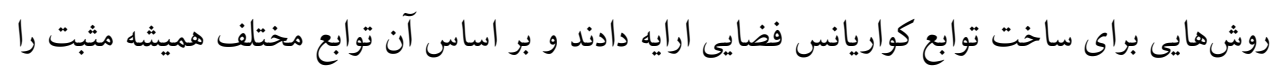
بهدست آوردند. بهعنوان مثال تابع برات سواتع

$$
C(h)=\sigma^{r} \exp \left(-\alpha h^{\beta}\right)
$$

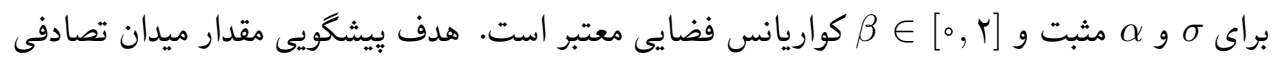

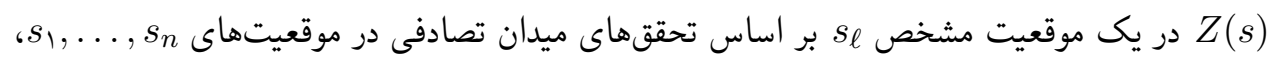
يعنى بردار مشاهدات $\left(z\left(s_{1}\right), \ldots, z\left(s_{n}\right)\right.$ 


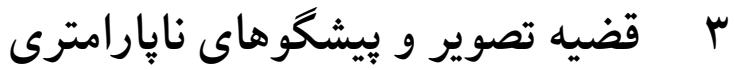

براى ميدان تصادفى (Z)

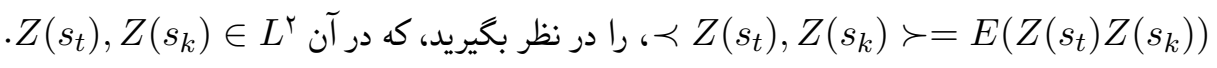

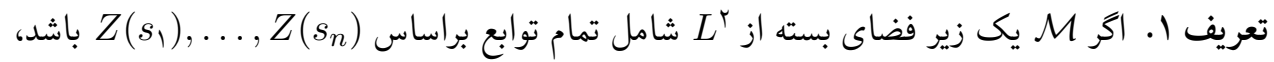
آنكاه تصوير (s) $Z$ روى

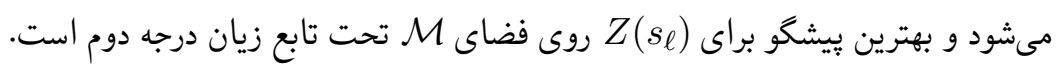

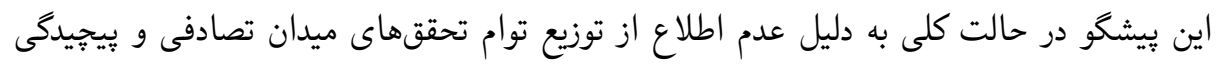

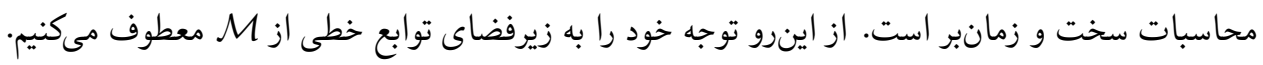

تعريف r. فرض كنيد

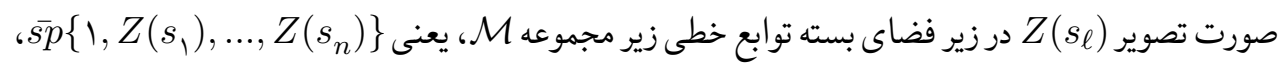
بهورت حل همزمان معادلات زير بهدست مى آيند.

$$
\sum_{i=\circ}^{n} a_{i} E\left(Z\left(s_{i}\right) Z\left(s_{j}\right)\right)=E\left(Z\left(s_{\ell}\right) Z\left(s_{j}\right)\right), \quad j=\circ, 1, \ldots, n .
$$

اميد شرطى، بر اساس قضيه تصوير، بهترين بيشكوى خطى براى (I)

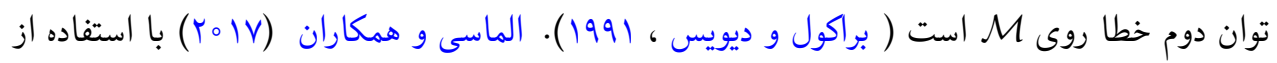

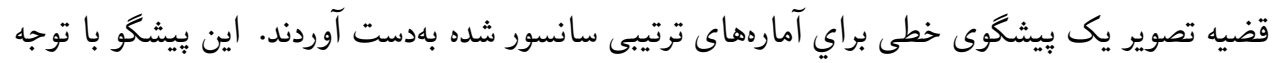

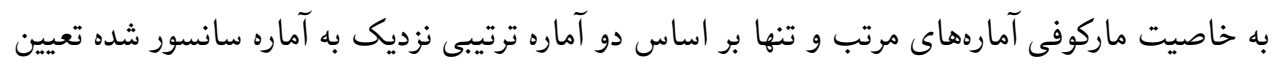

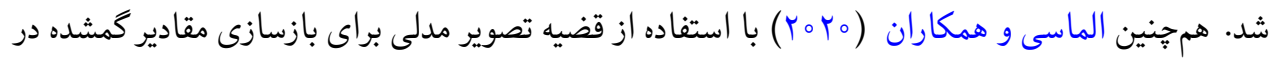

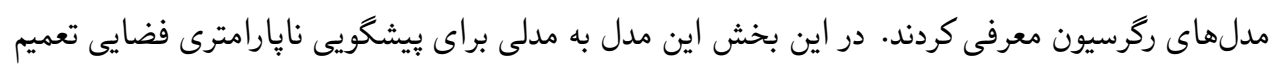

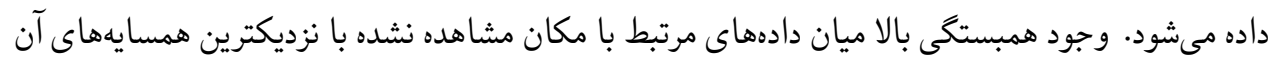

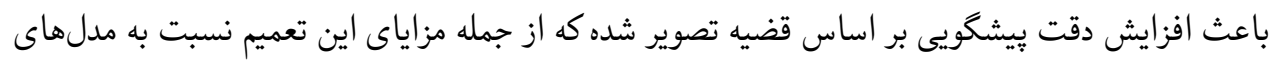

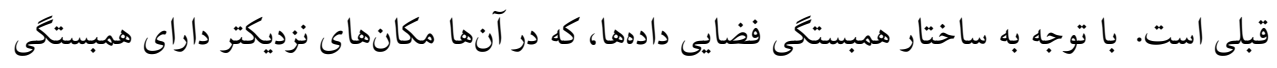

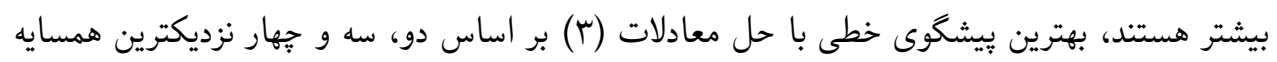
بددست مى آيد. 


\section{1. ب بهترين بيشكو بر اساس نزديكترين همسايكىها}

با توجه به ساختار همبستكى فضايى دادهها كه در آن نقاط نزديك داراى همبستكى بيشترى هستند، مىتوان

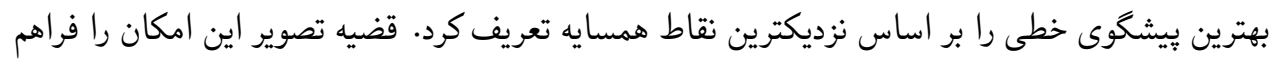

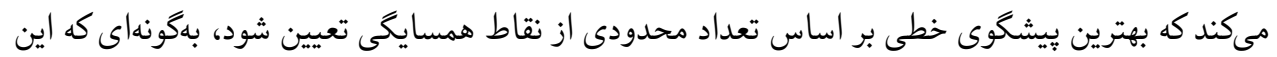

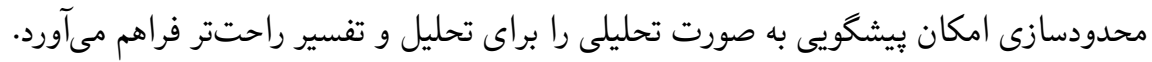

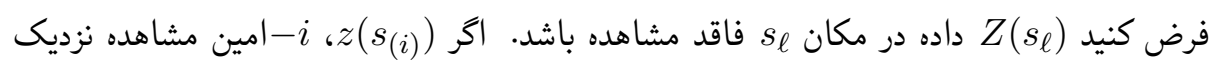

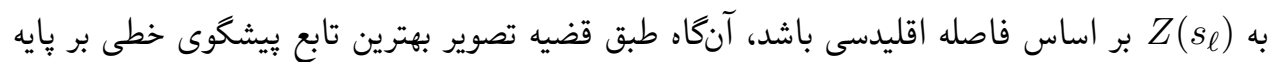

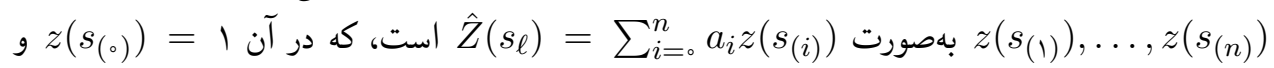
ضرايب a

$$
\sum_{i=\circ}^{n} a_{i} E\left(Z\left(s_{(i)}\right) Z\left(s_{(j)}\right)\right)=E\left(Z\left(s_{\ell}\right) Z\left(s_{(j)}\right)\right), \quad j=\circ, 1, \ldots, n
$$

$$
\begin{aligned}
& \text { فرع ا . بهترين بيشكوى خطى (S) } \\
& \text { و } z\left(s_{(\uparrow)}\right. \\
& \hat{Z}\left(s_{\ell}\right)=\sum_{i=0}^{\dagger} a_{i} z\left(s_{(i)}\right)
\end{aligned}
$$

$$
\begin{aligned}
& a_{\circ}=\mu\left(s_{\ell}\right)-a_{\uparrow} \mu\left(s_{(\uparrow)}\right)-a_{\digamma} \mu\left(s_{(\Upsilon)}\right)-a_{\digamma} \mu\left(s_{(\Upsilon)}\right)-a_{\digamma} \mu\left(s_{(\uparrow)}\right)
\end{aligned}
$$

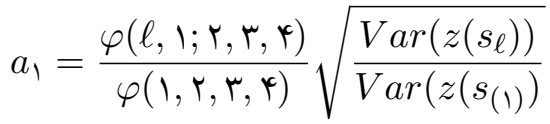

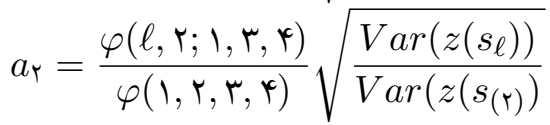

$$
\begin{aligned}
& a_{\boldsymbol{r}}=\frac{\varphi(\ell, \boldsymbol{r} ; \mathbf{1}, \boldsymbol{r}, \boldsymbol{\varphi})}{\varphi(\mathbf{l}, \boldsymbol{r}, \boldsymbol{r}, \boldsymbol{\varphi})} \sqrt{\frac{\operatorname{Var}\left(z\left(s_{\ell}\right)\right)}{\operatorname{Var}\left(z\left(s_{(\boldsymbol{r})}\right)\right.}}
\end{aligned}
$$

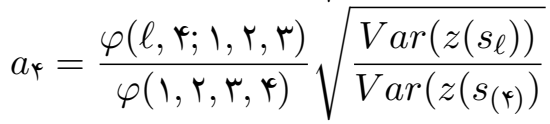




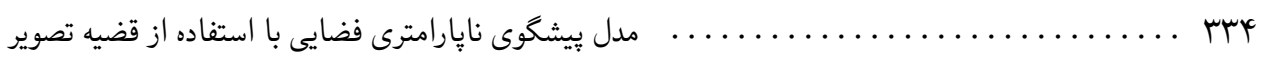

$$
\text { بهدست مى آيند، كه در آنها (·) ب توابعى از همبستكى فضايى بهصورت }
$$

$$
\begin{aligned}
& \varphi(1, r, r, r)=1-\rho_{l, r}^{r}-\rho_{l, r}^{r}-\rho_{l, \Psi}^{r}-\rho_{r, r}^{r}-\rho_{r, r}^{r}-\rho_{r, r}^{r}+\rho_{l, r}^{r} \rho_{r, r}^{r}
\end{aligned}
$$

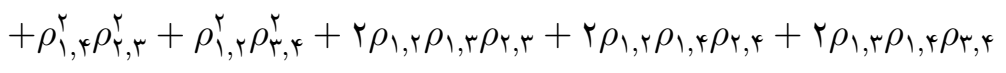

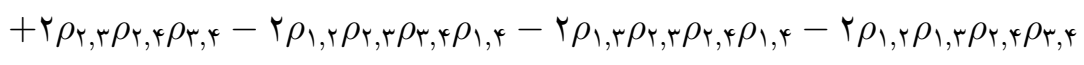

$\varphi(\ell, r ; k, s, m)=\rho_{\ell, r}\left(1-\rho_{k, m}^{r}-\rho_{k, s}^{r}-\rho_{s, m}^{r}+r \rho_{k, m} \rho_{k, s} \rho_{s, m}\right)$

$-\rho_{\ell, k}\left(\rho_{r, k}-\rho_{r, k} \rho_{s, m}^{r}-\rho_{r, s} \rho_{k, s}-\rho_{r, m} \rho_{k, m}+\rho_{r, m} \rho_{k, s} \rho_{s, m}+\rho_{r, s} \rho_{k, m} \rho_{s, m}\right)$

$-\rho_{\ell, s}\left(\rho_{r, s}-\rho_{r, s} \rho_{k, m}^{r}-\rho_{r, k} \rho_{k, s}-\rho_{r, m} \rho_{s, m}+\rho_{k, m} \rho_{r, m} \rho_{k, s}+\rho_{r, k} \rho_{k, m} \rho_{s, m}\right)$

$-\rho_{\ell, m}\left(\rho_{r, m}-\rho_{r, m} \rho_{k, s}^{r}-\rho_{r, s} \rho_{s, m}-\rho_{r, k} \rho_{k, m}+\rho_{r, k} \rho_{k, s} \rho_{s, m}+\rho_{r, s} \rho_{k, s} \rho_{k, m}\right)$

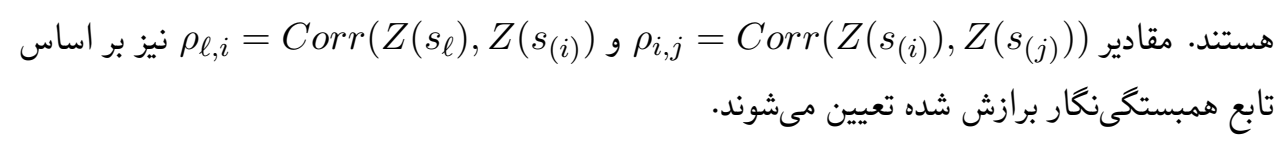

معادله بِيشكو و ضرايب آن براى نزديكترين دو همسايه و سه همسايه نيز بهطريق مشابه و از حل

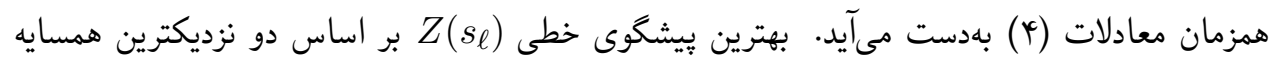
$z\left(s_{(1)}\right), z\left(s_{(\Upsilon)}\right)$

$$
\hat{Z}\left(s_{\ell}\right)=a_{\circ}+a_{\uparrow} z\left(s_{(\uparrow)}\right)+a_{\uparrow} z\left(s_{(\uparrow)}\right)
$$

است، كه در آن

$$
\begin{aligned}
& a_{\circ}=\mu\left(s_{\ell}\right)-a_{\uparrow} \mu\left(s_{(\curlywedge)}\right)-a_{\curlyvee} \mu\left(s_{(\curlyvee)}\right) \\
& a_{\uparrow}=\frac{\rho_{\ell, \uparrow}-\rho_{\ell, \Upsilon} \rho_{\ell, \Upsilon}}{1-\rho_{\uparrow, \Upsilon}^{r}} \sqrt{\frac{\operatorname{Var}\left(z\left(s_{\ell}\right)\right)}{\operatorname{Var}\left(z\left(s_{(\backslash)}\right)\right.}}, \\
& a_{\Upsilon}=\frac{\rho_{l, \Upsilon}-\rho_{\backslash, \Upsilon} \rho_{\ell, \uparrow}}{1-\rho_{\zeta, \Upsilon}^{r}} \sqrt{\frac{\operatorname{Var}\left(z\left(s_{\ell}\right)\right)}{\operatorname{Var}\left(z\left(s_{(\uparrow)}\right)\right.}}
\end{aligned}
$$




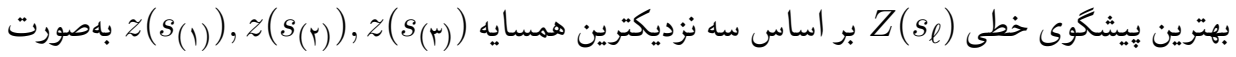

$$
\begin{aligned}
& \hat{Z}\left(s_{\ell}\right)=a_{\circ}+a_{\curlywedge} z\left(s_{(\uparrow)}\right)+a_{\Upsilon} z\left(s_{(\Upsilon)}\right)+a_{\Upsilon} z\left(s_{(\Upsilon)}\right)
\end{aligned}
$$

$$
\begin{aligned}
& a_{\circ}=\mu\left(s_{\ell}\right)-a_{\uparrow} \mu\left(s_{(\uparrow)}\right)-a_{\digamma} \mu\left(s_{(\Upsilon)}\right)-a_{\digamma} \mu\left(s_{(\Upsilon)}\right)
\end{aligned}
$$

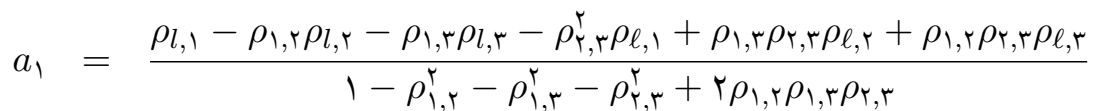

$$
\begin{aligned}
& \times \sqrt{\frac{\operatorname{Var}\left(z\left(s_{\ell}\right)\right)}{\operatorname{Var}\left(z\left(s_{(\curlywedge)}\right)\right.}}
\end{aligned}
$$

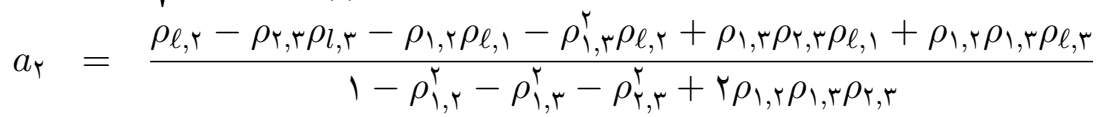

$$
\begin{aligned}
& \times \sqrt{\frac{\operatorname{Var}\left(z\left(s_{\ell}\right)\right)}{\operatorname{Var}\left(z\left(s_{(\Upsilon)}\right)\right.}}
\end{aligned}
$$

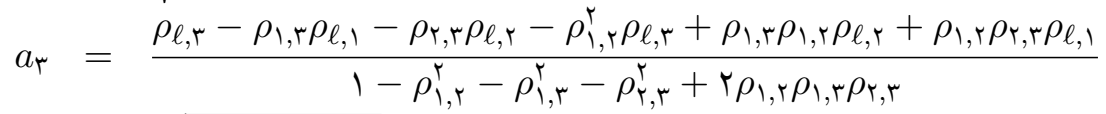

$$
\begin{aligned}
& \times \sqrt{\frac{\operatorname{Var}\left(z\left(s_{\ell}\right)\right)}{\operatorname{Var}\left(z\left(s_{(\mathrm{r})}\right)\right.}}
\end{aligned}
$$

\section{F مطالعه شبيهازى}

در اين بخش با استفاده از شبيهسازى، دقت مدلهاى معرفى شده در روابط (ه )، (4 ) و (V) مورد بررسى

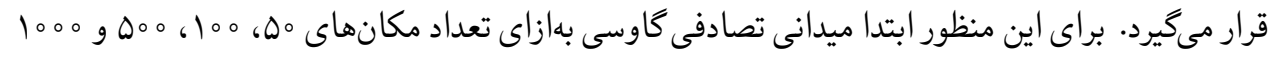

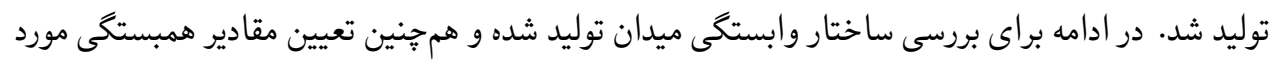

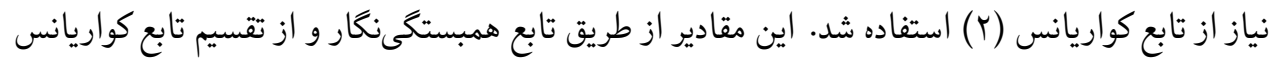

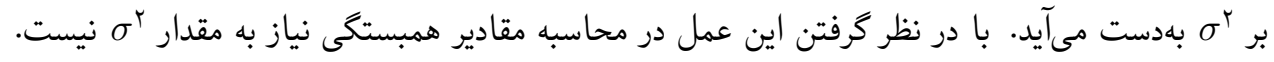

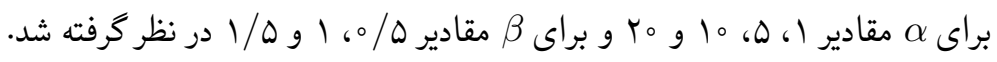

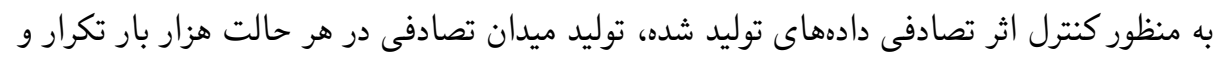

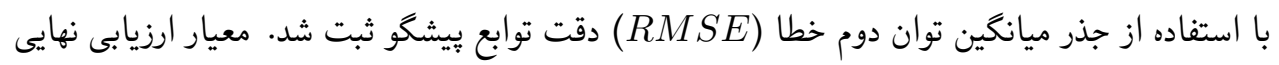




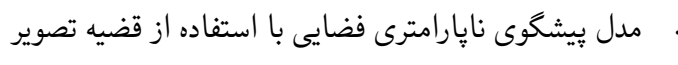

نيز ميانخين RMSE در اين هزار بار اجرا در نظر گرفته شد. در جدول ا مقادير بارامترها و ميانگين RMSE

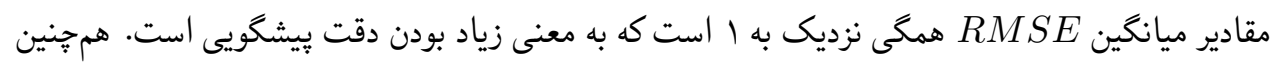

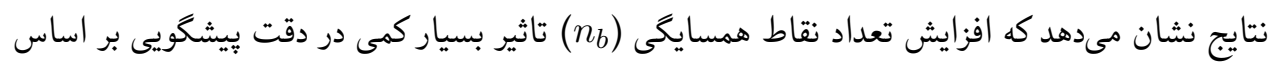

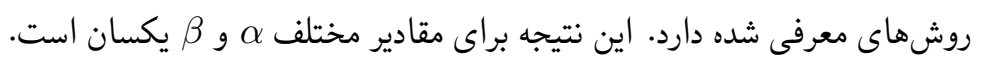

\section{ه مثال كاربردى}

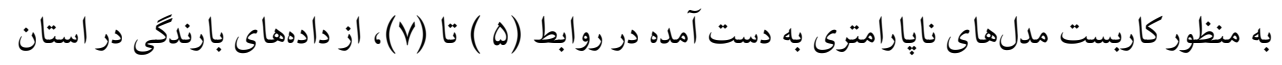

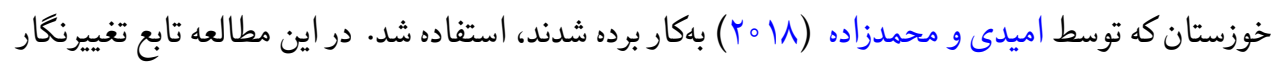

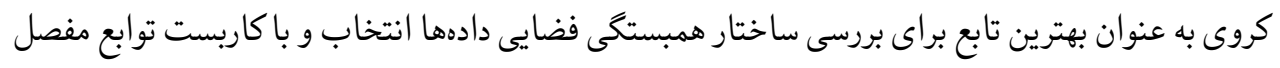

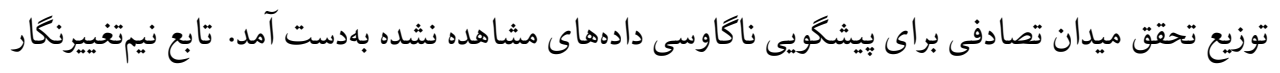
كروى بهصورت ${ }^{r}$

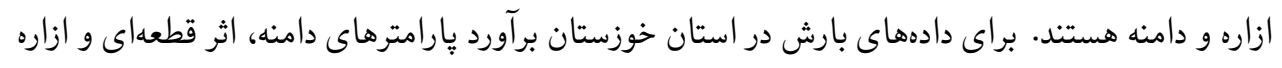

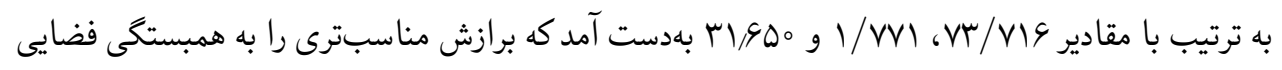

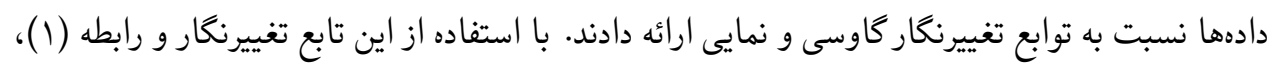

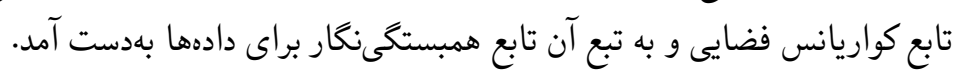
با در نظر كرفتن توابع همبستخىنگار و با به كاركيرى روابط (ه ) تا (V) مقدار يُيشكويى براى دادهها

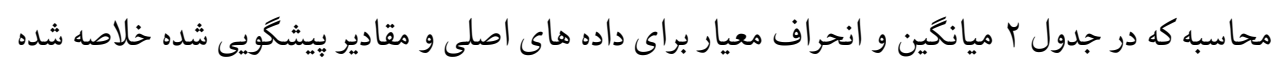

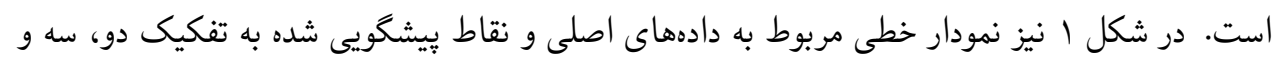

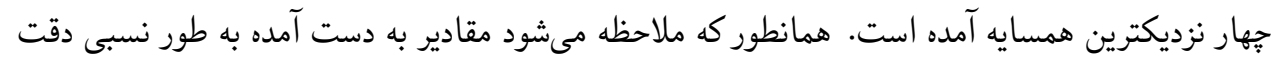

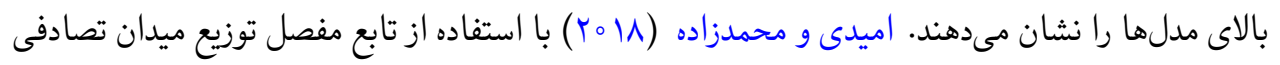

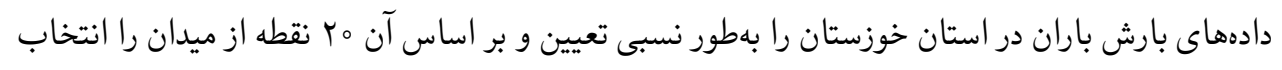

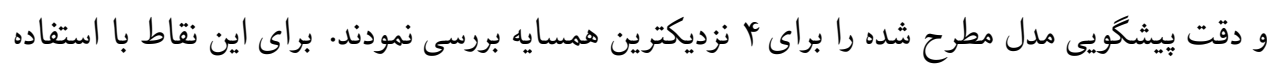

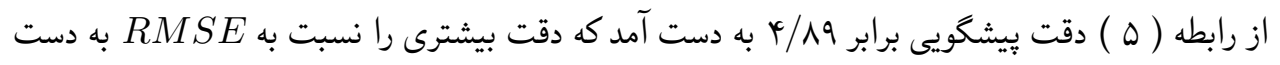

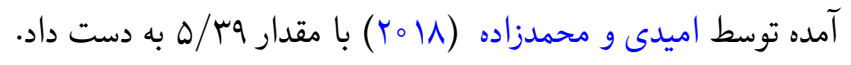


جدول ا. مقدار RMSE براى دادههاى شبيهسازى شده

\begin{tabular}{|c|c|c|c|c|c|c|}
\hline \multicolumn{4}{|c|}{$\alpha$} & \multirow[b]{2}{*}{$n_{b}$} & \multirow[b]{2}{*}{$\beta$} & \multirow[b]{2}{*}{$\mathrm{n}$} \\
\hline To & 10 & b & 1 & & & \\
\hline $1 / \circ \vee V 10$ &.$/ 99499$ &.$/ 90999$ & $T / \circ \Delta Y \Delta T$ & $r$ & & \\
\hline $1 / 00 V \mid F$ &.$/ 99499$ &.$/ 90910$ & 1/OTDYG & r & $\circ / \Delta$ & \\
\hline $1 / 00 V I r$ & -/994k4 &.$/ 90011$ & $1 / 01100$ & k & & \\
\hline - /qqMqY &.$/ 94 q 41$ & $1 / 19410$ & $1 / r$ TOSD & r & & \\
\hline $0 / 9941$ & - /qKTAK & $1 / 1 T \Delta Y \Delta$ & $1 /$ TOYGV & $r$ & 1 & $\Delta \circ$ \\
\hline -/99109 &.$/ 94010$ & I/IKYIT & I/MAVTA & c & & \\
\hline 1/OrgKY & $1 / \pi T V V \Lambda$ & I/TYOIT & $1 /$ rqV9D & r & & \\
\hline 1/OYYAV & $1 / R T \Delta Q Y$ & $1 / K k+40$ & $1 / 70109$ & $r$ & $1 / 0$ & \\
\hline $1 / 0 I V K Y$ & $1 / \pi / \Delta \Lambda$ & $1 / Y K \circ V I$ & $1 /$ TrVYF & r & & \\
\hline $1 / 000 Y \Lambda$ & $\circ / 94 M 9$ &.$/ 909 \mathrm{VA}$ & $1 / 0 \psi \Delta \| 1 \%$ & r & & \\
\hline $1 / 000 Y 1$ & -/qAvqr & -/9DYYG & $1 / 01 V 0 r$ & r & $\circ / \Delta$ & \\
\hline $1 / 0001 \mathrm{~V}$ & - /१४VQV & $0 / q 4 q 44$ & $1 / 010 \mathrm{VV}$ & t & & \\
\hline - /qkTqV & - /qraro & $1 / 010 V \lambda$ & 1/KTOGS & r & & \\
\hline ./94999 & 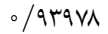 & $1 / 010 \pi r$ & $1 / 19441$ & r & 1 & 100 \\
\hline 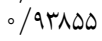 & ./940०० & $1 / 00 \% 19$ & $1 / 1 V Y 90$ & $r$ & & \\
\hline 1/० TDQY & 1/RTOGY & 1/YKAKT & $1 /$ r4914 & r & & \\
\hline $1 / 01901$ & $1 / r \mid \Delta k \psi$ & $1 / K K \mid r \circ$ & $1 /$ KYAYK & r & $1 / 0$ & \\
\hline $1 / 01 \vee 99$ & $1 / r \mid r \Delta \Delta$ & $1 / K Y \circ 99$ & I/KKMTI & $\varphi$ & & \\
\hline.$/ 99491$ & ./QGTT &.$/ 9 k 410$ & $1 / 04 \Delta F 1$ & r & & \\
\hline.$/ 994 V r$ &.$/ 90994$ & . /qrAvV & $1 / 0194$ & $r$ &.$/ 0$ & \\
\hline.$/ 99499$ & $\circ / 9 \Delta V V I$ & - /qTYYO & $1 / 00 \vee 99$ & $r$ & & \\
\hline - /qutra & - /qukar & $1 / 01 Y Y A$ & 1/1人सrq & r & & \\
\hline - /qTGTD & $. / 94<19$ & $1 / 009 V V$ & I/IOVVY & $r$ & 1 & 0,0 \\
\hline$\circ / 9 \mu \circ \gamma_{0}$ &.$/ 94190$ & $1 / 00197$ & $1 / 1 \% \Delta V R$ & i & & \\
\hline I/OIATV & $1 /$ rlkrq & $1 / 19411$ & 1/rkyar & r & & \\
\hline 1/०OVVY & I/TIYOY & $1 / 19441$ & $1 / r 41 \wedge 9$ & $r$ & $1 / 0$ & \\
\hline $1 / 0099 V$ & $1 / 41049$ & $1 / 10110$ & $1 /$ MYIYI & $\varphi$ & & \\
\hline - &.$/ 90119$ & $\circ / 94 \circ q 4$ & $1 / 00109$ & r & & \\
\hline.$/ ৭ \wedge \Lambda \Lambda \Delta$ & - /qYDQY & - arris & $1 / 00114$ & r &.$/ 0$ & \\
\hline 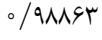 &.$/ 9 k 401$ & - /argkr & $1 / 000 r q$ & r & & \\
\hline - $/ 94100$ & $\circ / 94409$ & & $1 / 190 \wedge \mathrm{F}$ & r & & \\
\hline - /qugIV & $\circ / q r r \circ q$ & - /QAYYS & $1 / 1 r a \wedge r$ & r & 1 & 1000 \\
\hline$\circ / 9 \mu \circ \gamma_{0}$ &.$/ 9 M|F|$ & - /qVDGY & 1/TrVes & r & & \\
\hline $1 / 01 \Lambda \circ \mu$ & $1 / \pi 1109$ & $1 / 10 \wedge \Delta T$ & $1 / r 4109$ & r & & \\
\hline $1 / 00 \Delta 94$ & $1 / T 1091$ & $1 / 1 \% 4 \Delta V$ & $1 / r \mu \circ \Delta r$ & r & $1 / 0$ & \\
\hline 1/OOFAT & $1 / Y 10 \% \mu$ & $1 /\left|k \psi_{0}\right|$ & $1 / K Y_{0} F_{T}$ & r & & \\
\hline
\end{tabular}


·. . . مدل بيشكوى نايارامترى فضايى با استفاده از قضيه تصوير

MT
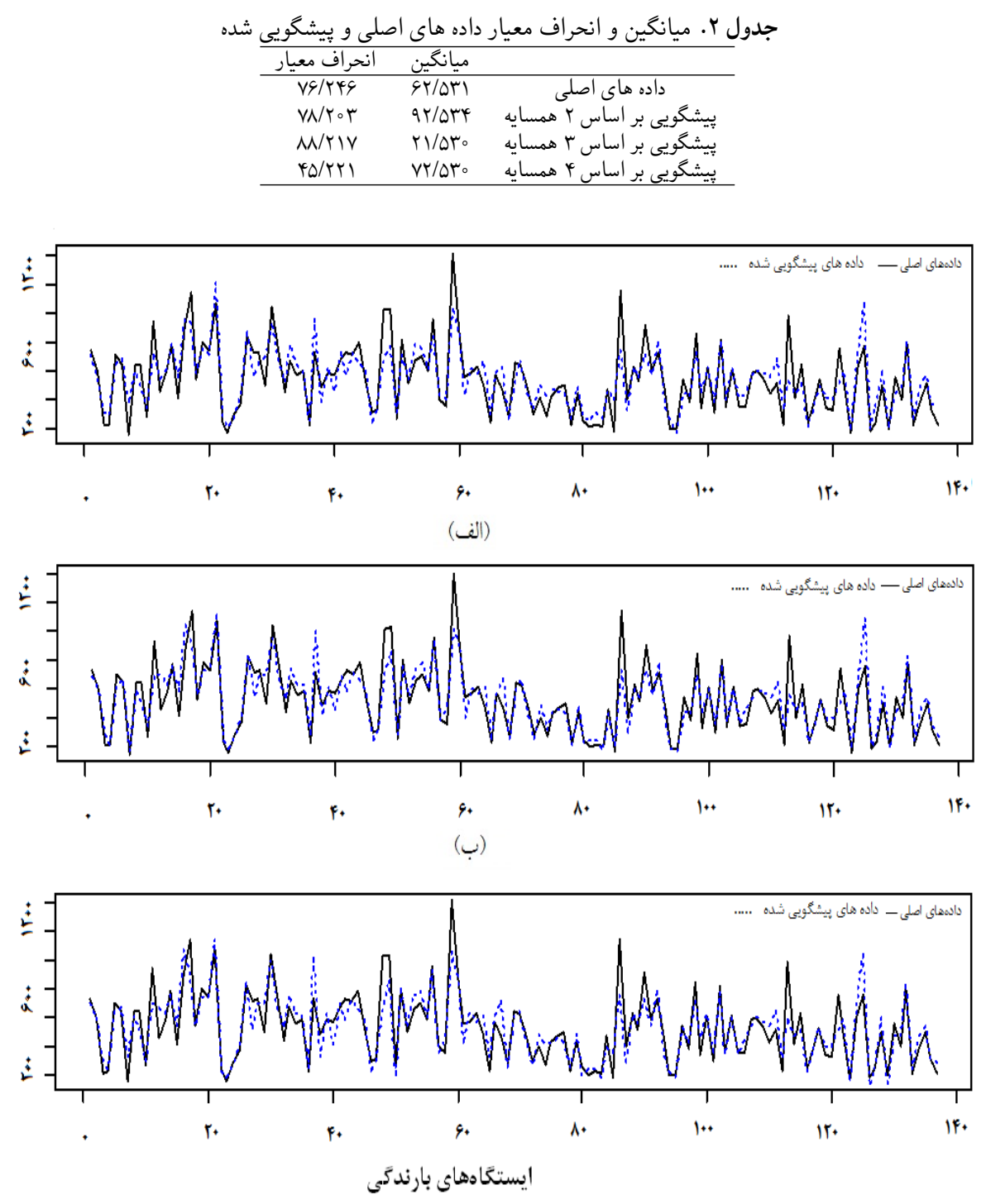

(ج)

شكل ا. نمودار دادهاى اصلى و نقاط بِيشكويى شده، الف-براى دو نزديكترين همسايه، ب-براى سه نزديكترين همسايه و ج-براى جهار نزديكترين همسايه 


\section{بحث و نتيجهگيرى}

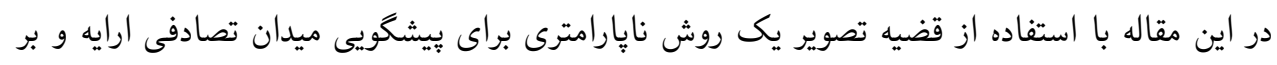

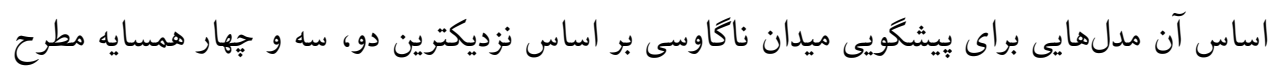

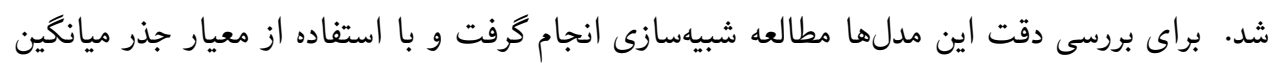

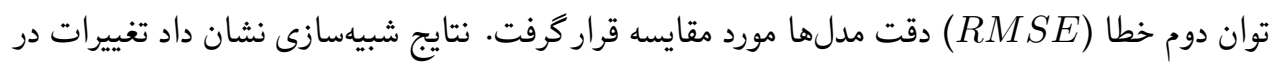

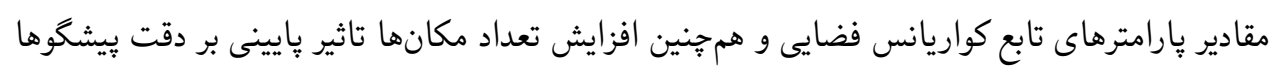

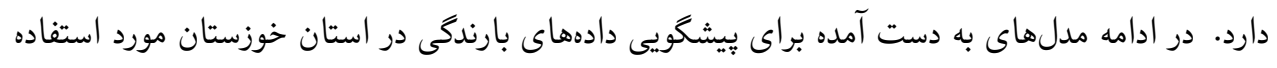

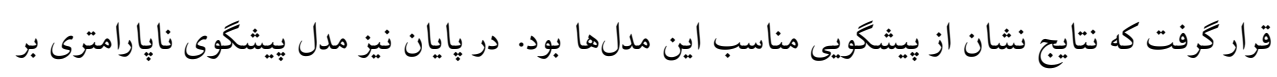

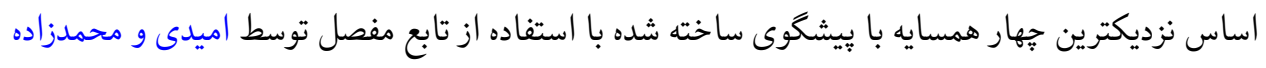

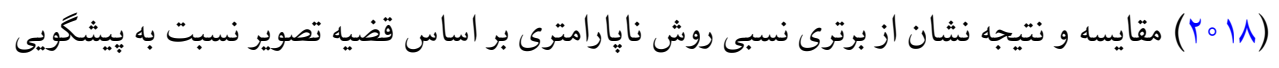

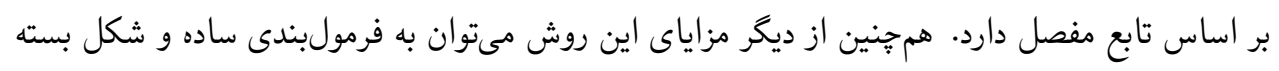

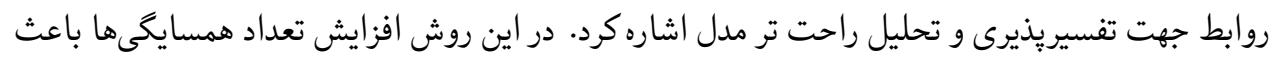

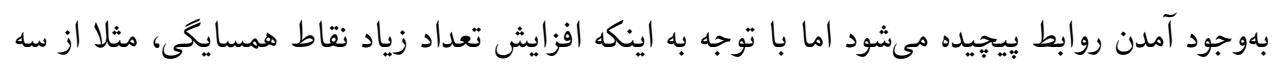

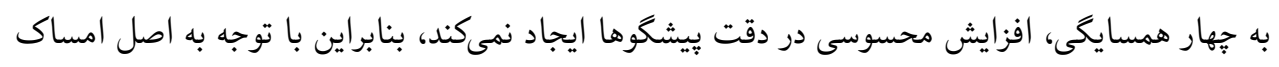

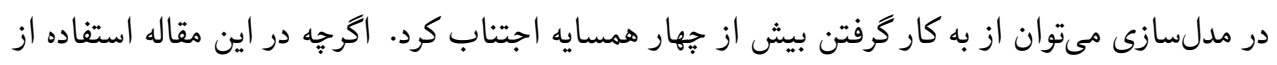

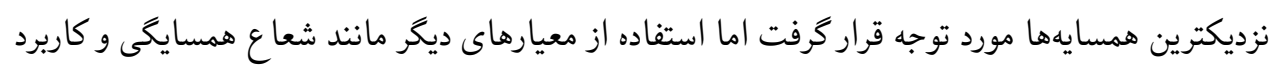

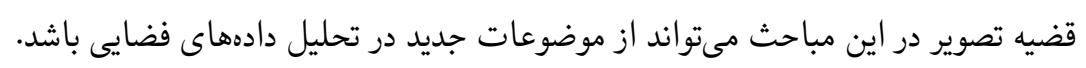

\section{تقدير و تشكر}

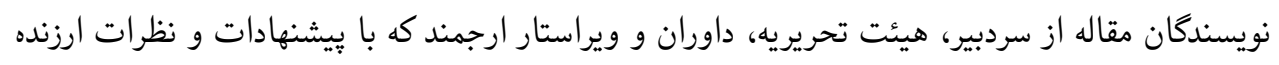

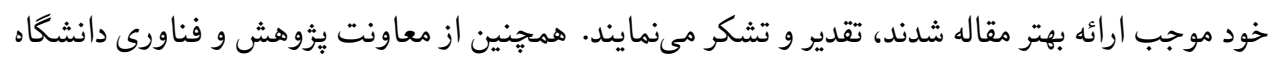
ايلام بهخاطر حمايت در انجام مقاله كمال تشكر و قدردانى را داند ماند. 


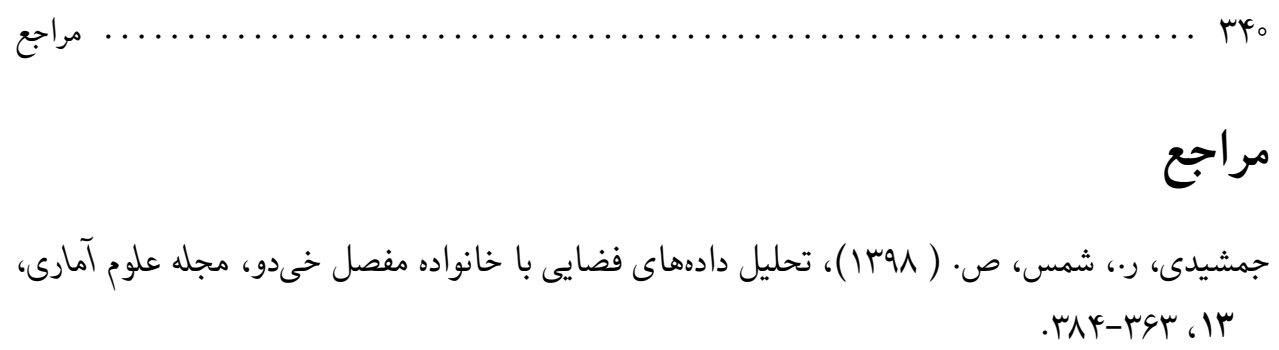

Almasi, I., Mohammadpour, A., and Mohammadi, M. (2017), Best Linear Unbiased Interpolation of Order Statistics. Communications in StatisticsSimulation and Computation, 46, 4161-4171.

Almasi, I., Mohsen Salehi, M. and Moradi, M. (2020), A New Algorithm to Impute the Missing Values in the Multivariate Case, JIRSS, 19, 133-143.

Arnold, B. C., Balakrishnan, N., and Nagaraja, H. N. (1992), A First Course in Order Statistics (Vol. 54). Siam.

Brockwell, P. J., Davis, R. A. (1991). Time Series: Theory and Methods, second ed., Springer Series in Statistics, Springer-Verlag, New York.

Cressie, N. (1993), Statistics for Spatial Data, John Wiley, New York.

Graler, B. and Pebesma, E., (2011), The Pair Copula Construction for Spatial Data, a New Approach to Model Spatial Dependency, Procedia Environmental Sciences, 7, 206-211.

Nelsen, R. B., (2006), An Introduction to Copulas, New York, Springer.

Omidi, M. and Mohammadzadeh, M. (2016), A New Method to Build SpatioTemporal Covariance Functions: Analysis of Ozone Data. Statistical Papers. 57, 689-703.

Omidi, M. and Mohammadzadeh, M. (2018), Spatial Interpolation Using Copula for Non-Gaussian Modeling of Rainfall Data, JIRSS, 17 , 165-179. 
Journal of Statistical Sciences, Autumn and Winter, 2021

Vol. 15, No. 2, pp 329-340

DOI: $10.29252 /$ jss.15.2.329

\title{
Construction a Non-parametric Prediction Model for Spa- tial Random Field Using Projection Theorem
}

\author{
Almasi ${ }^{1}$ ، I. ، Omidi، M. ${ }^{2}$ \\ ${ }^{1}$ Department of Statistics, Razi University, Kermanshah, Iran. \\ ${ }^{2}$ Department of Mathematics, Ilam University, Ilam, Iran.
}

\begin{abstract}
Identifying the best prediction of unobserved observation is one of the most critical issues in spatial statistics. In this line, various methods have been proposed, that each one has advantages and limitations in application. Although the best linear predictor is obtained according to the Kriging method, this model is applied for the Gaussian random field. The uncertainty in the distribution of random fields makes researchers use a method that makes the nongaussian prediction possible. In this paper, using the Projection theorem, a non-parametric method is presented to predict a random field. Then some models are proposed for predicting the nongaussian random field using the nearest neighbours. Then, the accuracy and precision of the predictor will be examined using a simulation study. Finally, the application of the introduced models is examined in the prediction of rainfall data in Khuzestan province.
\end{abstract}

Keywords: Nongaussian predictor, Random field, Kriging, Projection theorem.

Mathematics Subject Classification (2010): 62H11, 62M20. 